\title{
High-Efficiency Gold Fresnel Zone Plates for Multi-keV X-rays
}

\author{
S. Gorelick ${ }^{\mathrm{a}}$, J. Vila-Comamala ${ }^{\mathrm{a}}$, V. A. Guzenko ${ }^{\mathrm{a}}$, R. Barrett ${ }^{\mathrm{b}}$, \\ M. Saloméb, and C. David ${ }^{\mathrm{a}}$ \\ ${ }^{a}$ Paul Scherrer Institut, CH-5232 Villigen, Switzerland \\ ${ }^{b}$ ESRF, 6 rue Jules Horowitz, BP220, F-38043 Grenoble Cedex, France
}

\begin{abstract}
We report a direct e-beam writing process of Fresnel zone plates (FZPs) using thick layers of PMMA resist as electroplating molds. We used $100-\mathrm{kV}$ electron beam lithography to directly expose thick PMMA layers, which were later used as plating molds without intermediate etching steps. High-quality 500-nm- and 1- $\mu$ m-thick Au FZPs with outermost zone widths down to 50-nm and $70-\mathrm{nm}$, respectively, and with diameters up to $600 \mu \mathrm{m}$ were fabricated. In this paper we present the details of the optimized fabrication process, such as development times, developer, dose tables, and line shrinkages required to obtain the desired zone widths and gaps between the zones. The diffraction efficiencies of the fabricated FZPs were measured for a wide range of x-ray energies (2.8-13.2 keV) showing excellent values up to $65-75 \%$ of the theoretical maximum, reflecting the good quality of the FZPs. Spatially resolved diffraction efficiency measurements indicate the uniformity of the zone plates and lack of defects.
\end{abstract}

Keywords: Fresnel zone plate, hard x-rays, x-ray optics, electron beam lithography, Au electroplating, PMMA

PACS: 81.16.Nd, 41.50.+h, 07.85.Tt, 61.05.cp, 68.37.Yz, 87.59.-e, 42.82.Cr

\section{INTRODUCTION}

$\mathrm{X}$-ray microscopy has the potential for imaging thick samples with high resolution due to the high penetration power of x-rays and their short wavelength. To exploit fully the capabilities of $\mathrm{x}$-ray microscopy, it is necessary to efficiently control x-ray beams. Among a variety of techniques to manipulate the x-rays (e.g., for focusing or sample illumination), diffractive optical elements are some of the most promising. For instance, Fresnel zone plates (FZPs) can focus X-rays into spots smaller than $20 \mathrm{~nm}$ and even resolve sub-10-nm features [1]. The resolution performance of FZPs is roughly equal to the width of the outermost ring or "zone." To produce FZPs for high-resolution applications, high-resolution lithography methods are generally used. The method of choice is typically electron beam lithography (EBL), which is, in principle, capable of sub-10-nm patterning.

However, patterning FZPs by EBL with the smallest outermost zone width is not sufficient because the efficiency of FZPs - that is, the ratio of the focused to the incident intensity - depends on the height of the zones, as well as the material from which they are made and the photon energy. Ideally, the zones must be sufficiently tall to provide a phase-shift close to $\pi$ for the best diffraction efficiency. For soft $\mathrm{x}$-rays the required heights are typically of the order of a few hundreds of nm, while for the hard $x$-rays the optimal zone height would be several $\mu \mathrm{m}$ even for high- $Z$ materials with high refractive index (e.g., Au). Thus, to provide high-efficiency focusing into a small spot, FZPs must have nanostructured zones with extremely high aspect ratios. The EBL is, therefore, followed by post-processing steps, e.g., several etchings and electroplating [2-5], to transfer the low-aspect-ratio structures produced by EBL in thin layers of photoresist into taller and metallic nanostructures.

The fabrication of FZPs with high-aspect-ratio zone can, therefore, be challenging. A much faster and more reliable approach is to use $100-\mathrm{keV}$ electron beam lithography to directly write high-aspect-ratio nanostructures in thick layers of resist, which can be used as a mold for electroplating to produce metallic FZPs [6,7]. High-energy $100-\mathrm{keV}$ electrons are able to penetrate several $\mu \mathrm{m}$ into the resist with little scattering, which makes them suitable for exposure of high-aspect-ratio nanostructures in polymethyl-methacrylate (PMMA) resist. FZPs made from Au were produced by filling the PMMA molds with Au by electroplating.

The 10th International Conference on X-ray Microscopy

AIP Conf. Proc. 1365, 88-91 (2011); doi: 10.1063/1.3625311

(C) 2011 American Institute of Physics 978-0-7354-0925-5/ $\$ 30.00$ 
The efficiencies of several fabricated FZPs were measured in a wide range of energies (2.8-13.2 keV). The measured efficiencies are relatively close to the theoretical maximum efficiency values, reflecting good quality of the FZPs.

\section{FABRICATION PROCESS}

The fabrication process started by vapor-coating $\mathrm{Si}_{3} \mathrm{~N}_{4}$ membranes with $\mathrm{Cr} / \mathrm{Au} / \mathrm{Cr}$ plating base $(5 / 20 / 5 \mathrm{~nm})$. The upper $\mathrm{Cr}$ layer was necessary to promote the adhesion of PMMA nanostructures, which adhered weakly to the Au surface. Resist layers were spin-coated on the membranes to yield 540-nm- or 1.1- $\mu$ m-thick layers. The exposure of patterns was performed on a 100-keV electron beam writer (Vistec EBPG 5000plus). Exposed line dimensions were reduced by 30-40 $\mathrm{nm}$ with respect to the target line width at high doses, such that after the development, the target width of the lines was achieved. The exposure doses were empirically determined as a function of the line width (Fig. 1). More detail on the optimization of the dose-shrinkage-development parameters can be found elsewhere [8]. Finally, the chips were developed in IPA:water (7:3) mixture for $10 \mathrm{~s}$ and $20 \mathrm{~s}$ for $540-\mathrm{nm}-$ and $1.1-\mu \mathrm{m}$-thick PMMA layers, respectively; after etching the upper $\mathrm{Cr}$ layer, the patterns were transferred into Au by electroplating.
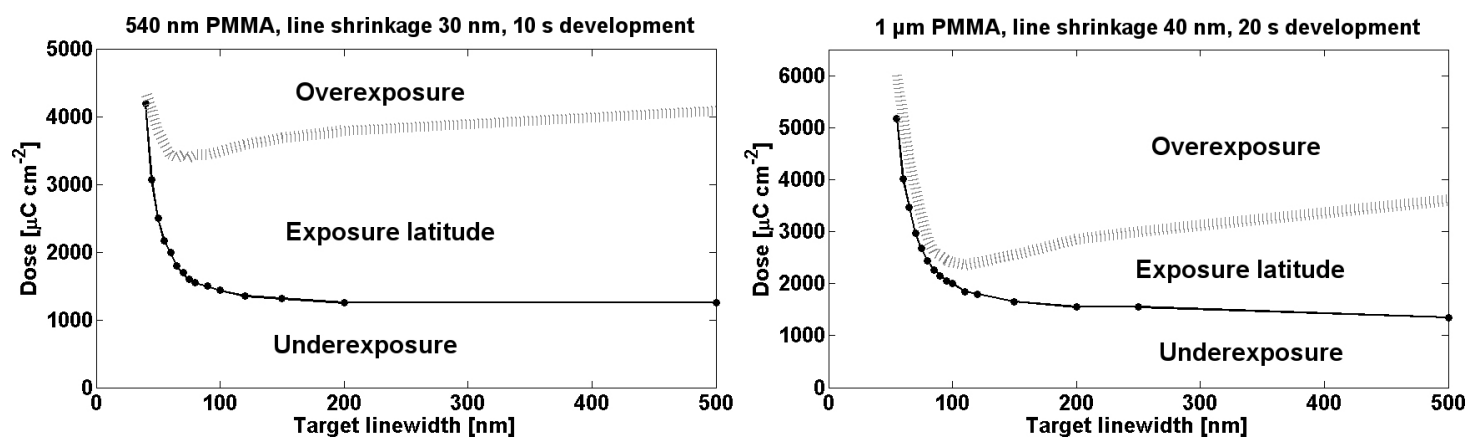

FIGURE 1. Area doses and exposure latitude for gratings of different periods exposed in PMMA for 30-nm and 40-nm line shrinkages in (a) 540-nm-thick PMMA and (b) 1.1- $\mu$ m-thick PMMA, respectively

Figure 2 shows some of the fabricated FZPs. The structures are uniform, and the overall quality of the devices is exceptionally good. During the exposure of the zone plates, we typically varied the doses such that the doses remained within the exposure latitudes (Fig. 1). We tried to keep the exposure doses as close as possible to the clearing doses, that is, to the interface between underexposure and the exposure latitude (Fig. 1); however, we noticed that often the patterns in PMMA were not fully developed, presumably due to uncertainties in the developer concentration and its temperature. By using higher doses, typically 10-15\% above the clearing doses, we avoided this problem leading to yields close to $100 \%$. In addition, rather than exposing continuous lines in PMMA, we exposed interrupted lines that were later translated into interrupted Au zones in FZPs. This segmentation was necessary to improve the mechanical stability of PMMA high-aspect-ratio lines, which tended to collapse during the drying process due to the action of capillary forces. To assess the quality of the fabricated zone plates, we measured their diffraction efficiencies. This information is useful for understanding the effects of processes parameters, such as exposure doses and the line segmentation, on the performance of the FZPs in order to further optimize the fabrication process and, consequently, fabricate the most efficient $\mathrm{x}$-ray diffractive lenses.

\section{DIFFRACTION EFFICIENCY MEASUREMENTS}

The diffraction efficiency measurements for energies above $6 \mathrm{keV}$ were performed at the ID06 beamline of the European Synchrotron Radiation Facility (ESRF). The measurements for energies below $6 \mathrm{keV}$ were performed at the ID21 beamline, ESRF. The efficiency measurement was performed by scanning a pinhole through the first-order focal spot of a zone plate that was illuminated through an aperture having exactly the same diameter as the zone plate. The focused intensity was calculated by subtracting the background signal caused by $0^{\text {th }}$-order radiation. Next, both the FZP and pinhole were removed, and the intensity of the incident beam was measured. The diffraction efficiency was estimated as a ratio of the focused to incident intensity. The measured efficiencies for three different types of the zone plates are presented in Fig. 3. 

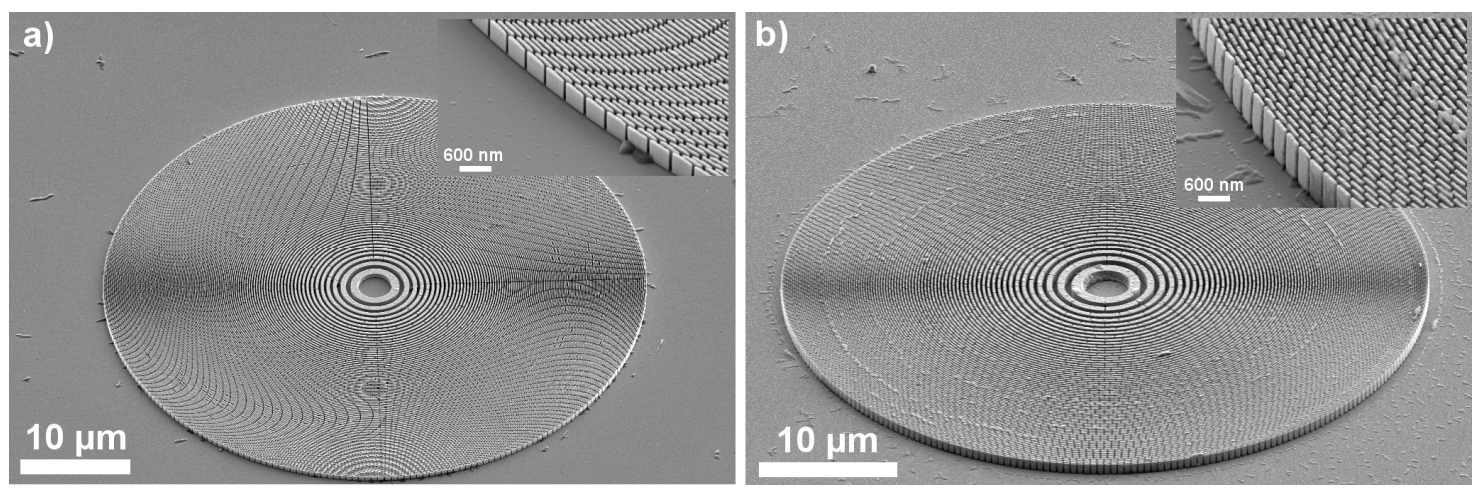

FIGURE 2. Scanning electron microscope images of $50 \mu \mathrm{m}$ in diameter (a) 0.5 - $\mu \mathrm{m}$-thick (tilt $45^{\circ}$ ) and (b) 1 - $\mu \mathrm{m}$-thick (tilt $55^{\circ}$ ) Fresnel zone plates (FZPs) with 50-nm and 70-nm outermost zone, respectively. The FZPs were fabricated by electroplating Au in PMMA molds that were produced by directly writing into the resist with $100-\mathrm{keV}$ electrons. The insets present magnified outer regions of the structures.

Generally, the efficiencies are above $60-80 \%$ of the theoretical maximum value [9] for 500-nm-thick FZPs, approaching $75-80 \%$ for the $1-\mu \mathrm{m}$-thick FZPs with a 100 -nm outermost zone. The discrepancy between the theoretical and measured values cannot solely be explained by the presence of the interruption of the zones. The phase-shifting area "lost" to the interruptions accounts for only $3 \%, 5.5 \%$, and $7.2 \%$ of the measured FZPs with 100 $\mathrm{nm}, 70-\mathrm{nm}$, and 50-nm outermost zones, respectively. The main factor contributing to the reduction of the diffraction efficiency is lower density of electroplated $\mathrm{Au}$ of $17.2 \mathrm{~g} \mathrm{~cm}^{-3}$ compared to $19.3 \mathrm{~g} \mathrm{~cm}^{-3}$ for bulk Au (AUTRONEX-CC electrolyte data sheet, Enthone Corp.). This density decrease results in reduced phase-shift of incoming X-ray beam and consequently to the reduced efficiency. For $1-\mu \mathrm{m}$-thick FZPs, the corresponding decrease in the efficiency is e.g., $13.25 \%$ for 8 -keV photons [8], while for 500-nm-thick FZPs the decrease is typically larger, e.g., $18.6 \%$ for $8-\mathrm{keV}$ photons and even larger for higher $\mathrm{x}$-ray energies [8]. Thus the zone segmentation and the reduced density of electroplated Au together result in $15-25 \%$ loss of the diffraction efficiency, while the remaining $10-15 \%$ loss can be attributed to the uncertainty in the zone width, which can vary to up to $10 \%$ from the optimal line width [7]. FZPs that were exposed at increased doses had typically broadened zones, and their measured efficiencies were systematically lower than those of the FZPs that were exposed at lower doses (not shown). We, therefore, conclude that using the lower exposure doses in our FZPs fabrication process is optimal. Further research and development of electroplating bath chemistry and plating conditions is required to optimize the density of the deposited $\mathrm{Au}$, which can lead to the fabrication of FZPs with efficiencies approaching the theoretical maximum values.
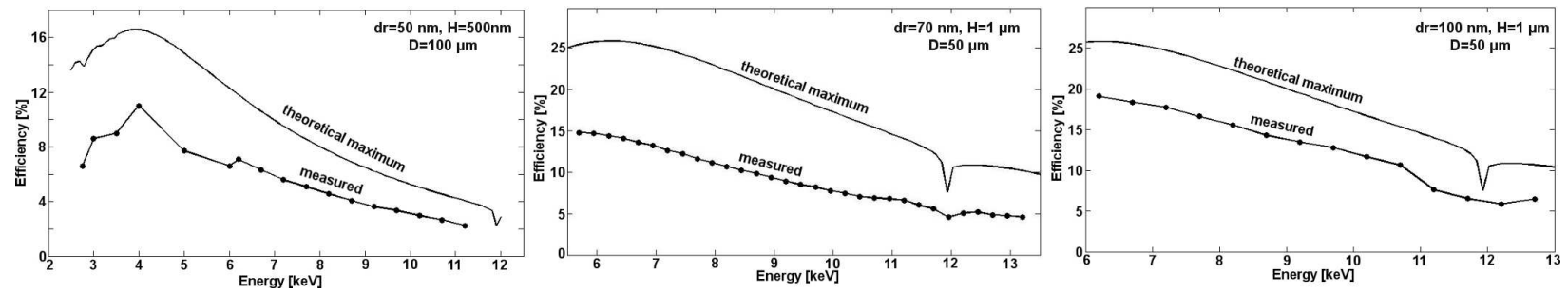

FIGURE 3. Diffraction efficiency (first order) of various Fresnel zone plates measured in a wide range of x-ray energies. The measured values are compared with the theoretically calculated efficiencies.

\section{SPATIALLY RESOLVED EFFICIENCY MEASUREMENTS}

Spatially resolved efficiency measurement of a 500-nm-thick, 100- $\mu$ m-diameter FZP with an outermost zone of $50 \mathrm{~nm}$ was performed at $6.2 \mathrm{keV}$ photon energy. The measurement was performed by illuminating the FZP through a 5- $\mu \mathrm{m}$ pinhole and selecting the focused intensity through another 5- $\mu \mathrm{m}$ pinhole positioned in the focus of the FZP (Fig. $4(a, b)$ ). By scanning the upstream pinhole, the map of local diffraction efficiencies was collected. The result of such a measurement is presented in Fig. 4(c). The profile of the local diffraction intensities is remarkably uniform, which indicates a lack of defects in the FZP. The local diffraction intensity decreases toward the edges of the FZP. This is consistent with the design of the FZP, i.e., radially increasing density of the zone interruptions, which was 
necessary to support thin outermost PMMA zones. In the studied FZP, the phase-shifting area "lost" due to the interruption in the outermost zone accounts for $21 \%$ of the zone area. While for the inner zones, where a few interruptions are found, the local diffraction efficiency is 0.088 , the local efficiency of the outermost zones is $25 \%$ lower at 0.066 . This is in good agreement with the loss of efficiency associated with the zone interruptions.
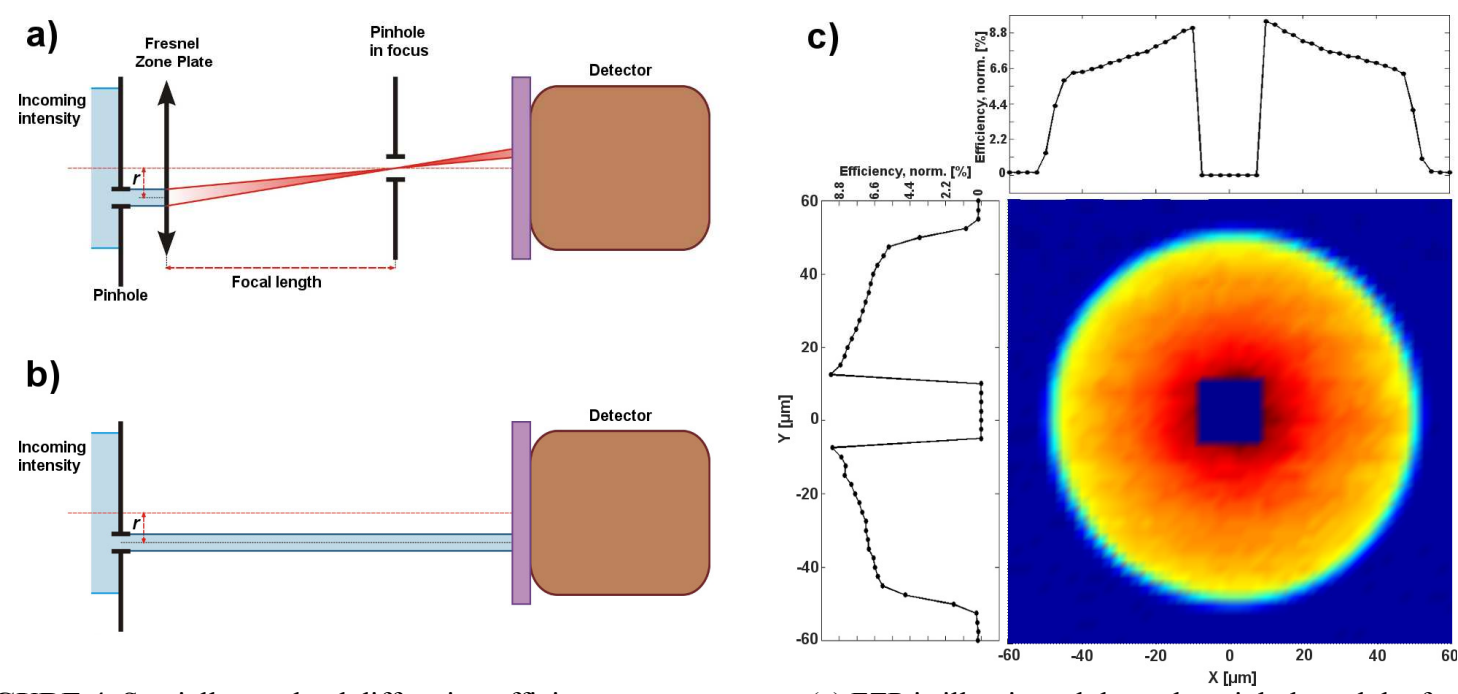

FIGURE 4. Spatially resolved diffraction efficiency measurement. (a) FZP is illuminated through a pinhole and the focused intensity is filtered by an order selecting aperture (OSA) in the focus of the FZP. (b) FZP and the OSA are removed and the incoming intensity is measured. (c) Spatially resolved diffraction efficiency of a $500 \mathrm{~nm}$-thick, 100- $\mu$ m-diameter FZP with an outermost zone of $50 \mathrm{~nm}$ at $6.2-\mathrm{keV}$ photon energy.

\section{CONCLUSION}

We developed a fast and reliable fabrication method for high-quality FZPs for multi-keV x-rays. The diffraction efficiencies of several FZPs were measured in a wide range of x-ray energies to assess the quality of the FZPs and the influence of various fabrication parameters on the diffraction efficiency. The measured diffraction efficiencies are $60-80 \%$ of the theoretical maxima. The main cause of the efficiency loss as compared to the theoretical maxima is the density of electroplated $\mathrm{Au}$. The effect of buttressing of polymer mold was manifested in decreased local efficiency of the areas in the FZP having higher density of buttressing. The overall local diffraction efficiency is uniform, which indicates a good quality of the FZP and lack of defects.

\section{ACKNOWLEDGMENTS}

We are thankful to M. Bednarzik, B. Haas, A. Weber, E. Deckhardt, and A. Lücke for assistance during substrate preparation. This work has been carried out under the auspices of Collaborative Project NFFA-Nanoscale Foundries and Fine Analysis, contract No. 212348, of the Seventh EU Framework Programme. The research leading to these results has also received funding from the European Community's Seventh Framework Programme (FP7/2007-2013) under grant agreement 226716.

\section{REFERENCES}

1. J. Vila-Comamala et al., Ultramicroscopy 109, 1360 (2009).

2. G. Schneider, T. Schliebe, and H. Aschoff, J. Vac. Sci. Technol. B 13, 2809 (1995).

3. K. Jefimovs et al., Microelectron. Eng. 841467 (2007).

4. Y. Feng et al., J. Vac. Sci. Tech. B 25, 2004 (2007).

5. M. Lindblom, H. Hertz, and A. Holmberg, Microelectron. Eng. 84, 1136 (2007).

6. Y.T. Chen et al., Nanotechnology 19395302 (2008).

7. S. Gorelick et al., Microelectron. Eng. 87, 1052 (2010).

8. S. Gorelick, J. Vila-Comamala, V.A. Guzenko, C. David, Nanotechnology 21, 295303 (2010).

9. B. L. Henke, E. M. Gullikson, and J. C. Davis, Atom. Data Nucl. Data 54(2), 181 (1993). Also available from http://henke.lbl.gov/optical_constants 